\title{
Intralist stimulus similarity and facilitation of paired-associate learning by practice distribution
}

\author{
MUKUL K. DEY,' Mount Allison \\ University, Sackville, N.B., Canada
}

Two degrees of intralist stimulus similarity were arranged by using two sets of CCC trigrams in the auditory mode. The same three letters were combined in different temporal patterns in one set, while the other set was composed of entirely different letters. These two conditions were factorially combined with three intertrial intervals: 2,30 , and $60 \mathrm{sec}$. A significant improvement in learning with increase in intertrial interval was found under the former but not under the latter condition. This result shows that paired-associate learning may not be facilitated by practice distribution unless there is sufficient intralist stimulus similarity and thus supports the writer's interpretation of the interference basic to distributed-practice efficacy. Further, an analysis of the intrusion errors elicited under the high-similarity condition reveals that more overt transfer of response occurred between two trigrams when both had the same letter at the first or third position than when they had the same letter at either the middle or no position. This fact is interpreted by a hypothesis based upon the concepts of perseverative stimulus trace and generalization between stimulus compounds.

The writer has developed a theory that interprets the interference in PA learning as a temporally labile inhibitory potential generated by the conflict of simultaneously evoked response tendencies (Dey, 1966). A principal prediction derived from this theory is that distribution of practice (DP) and intralist stimulus similarity (ISS) influence PA leaming in an interactive pattern. This prediction has been successfully tested in a previous study by the writer (Dey, 1966) which has revealed a joint influence of ISS and interunit interval (IUI), so that with higher values of the former, increase in the latter leads to greater facilitation. Supporting evidences have also been reported by Underwood \& Ekstrand (1967) who manipulated DP by varying intertrial interval (ITI). A further test of this prediction was arranged in the experiment reported in this paper by using the same stimulus terms as used in the earlier study (Dey, 1966) but manipulating DP through the variation of ITI.

\section{DESIGN, SUBJECTS, AND MATERIALS}

With ISS varied in two ways through the use of a low-similarity (LS) and a high-similarity (HS) stimulus series and ITI varied in terms of 2,30 , and $60 \mathrm{sec}$, the experiment was given a 2 by 3 factorial design. One hundred and eight sophomore and junior students derived from the writer's classes were assigned randomly to the six groups of equal size that are designated as LS-2, HS-2, LS-30, HS-30, LS-60, and HS-60. The Ss' mean age was 22.6 years in the range from 18 to 29.5 years. The male-female ratio was $3: 2$ in every group.

The HS stimuli are six $\mathrm{CCC}$ trigrams derived by combining three letters in all possible sequences: GKT, GTK, TKG, TGK, KTG, and KGT. The LS stimuli are six CCC trigrams with no common letter: HLF, BJX, SQZ, RMY, NDC, and VWP. The six three-letter words adopted as the $R$ terms in all lists are ham, fig, ray, box, jet, and cup. Counterbalancing the factor of S-R pairing, so that each stimulus was once paired with every $R$ term, six PA lists were derived for each degree of ISS.

\section{APPARATUS AND PROCEDURE}

The apparatus were two Tandberg tape recorders with solenoid start-stop switch, one Leitz Pradovit-Color slide projector with remote control and automatic return mechanism, one Scientific Prototype sound-operated relay, three Hunter interval timers, and two Lehigh Valley Electronics pulse-formers.

One of the tape recorders and the slide projector were used for presenting the stimuli and the $\mathbf{R}$ terms, respectively. The stimuli appeared at invervals of 9 sec. After $4 \mathrm{sec}$ from the start of every stimulus presentation, the $\mathbf{R}$ term was exposed for $2.5 \mathrm{sec}$. Assuming that the time consumed in the presentation of the entire stimulus trigram was approximately $1.5 \mathrm{sec}$, the anticipation interval was roughly $2.5 \mathrm{sec}$, and making some allowance for the relay times, IUI may be estimated as 2 sec. Three hour-long tapes were prepared for each stimulus series, repeatedly recording three 24-item sequences, one on each tape. The three stimulus sequences were derived in such a manner that the use of each in conjunction with the same six sequences of 24 slides-4 for each word-would furnish the desired counterbalancing of $S \cdot R$ pairing.
The instruction was the same as used by Dey (1966) plus some explanatory remarks concerning the rest-interval activity. For all groups, practice was carried on up to the criterion of $6 / 6$, which means the first perfect anticipation of all six $R$ terms on a trial. The groups receiving 2-sec ITI practiced continuously, as in their case ITI and IUI were of the same length. The groups receiving 30 and $60-\mathrm{sec}$ ITIs discriminated between tones spaced an octave apart as the rest-interval activity. The second tape recorder, which presented the pairs of tones at intervals of $9 \mathrm{sec}$, was instantly started as the first tape recorder (delivering stimuli) was stopped at the end of every trial. The two tones in each pair that were of $1-\mathrm{sec}$ duration each were separated by a .5-sec interval. Reports of judgment were made by $S$ always with reference to the second tone. The 30 - and 60-sec ITIs were, respectively, filled by three and six judgments each.

\section{RESULTS}

The mean trials required by the six groups to reach the criteria $1 / 6$ through $6 / 6$ are portrayed in the curves of Fig. 1. The divergence between the HS and LS . groups of curves appear to be increasing steadily over the practice stages. Further, while the curves in the LS group do not display consistent difference, those in the HS group clearly suggest facilitative influence from both 30 - and 60 -sec ITIs, especially at the later stages of practice.

The above observations are well supported by the results of a comparison of the mean trials to criterion 6/6 (Table 1) by a 2 by 3 analysis of variance. The large

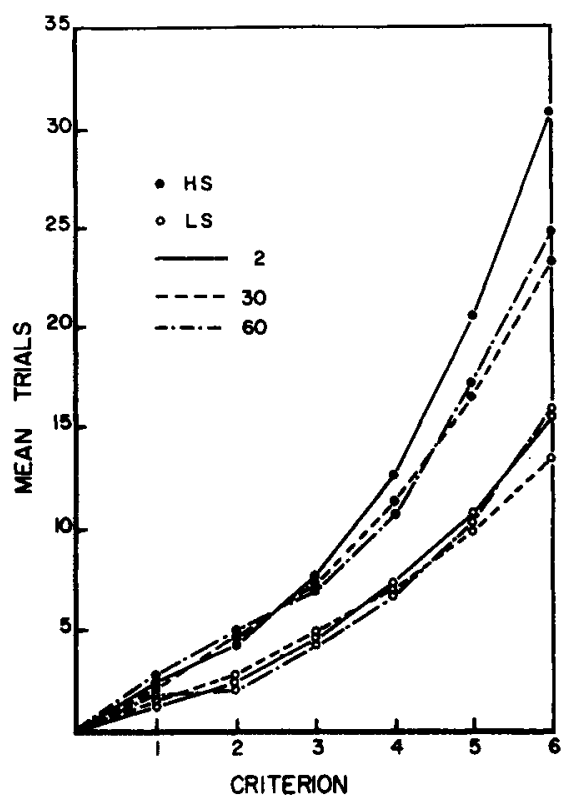

Fig. 1. Trials-to-criterion learning curves for the six groups. 
Table 1

Group Means and Standard Deviations of Trials to Criterion 6/6, Total Intrusions, and Per-Trial Intrusions

\begin{tabular}{lcccccr}
\hline & \multicolumn{2}{c}{ Trials to Criterion 6/6 } & \multicolumn{2}{c}{ Total Intrusions } & \multicolumn{2}{c}{ Intrusions Per Trial } \\
\cline { 2 - 6 } Group & Mean & SD & Mean & SD & Mean & SD \\
\hline LS-2 & 15.5 & 5.33 & 27.00 & 13.45 & 1.77 & .97 \\
LS-30 & 13.44 & 3.99 & 29.33 & 12.87 & 2.09 & 1.18 \\
LS-60 & 15.72 & 4.55 & 32.33 & 16.92 & 2.15 & 1.38 \\
HS-2 & 30.5, & 10.48 & 63.90 & 30.43 & 1.99 & 1.04 \\
HS-30 & 23.22 & 8.34 & 52.77 & 24.93 & 2.24 & .93 \\
HS-60 & 24.78 & 9.6 & 46.39 & 35.9 & 1.72 & 1.21 \\
\hline
\end{tabular}

Table 2

A Breakdown of Mean Intrusion Frequencies (see text for explanation)

\begin{tabular}{lrrrrr}
\hline $1-2-3$ & $1-3-2$ & $2-1-3$ & $3-2-1$ & $3-1-2$ & $2-3-1$ \\
\hline KTG & 2.07 & 1.82 & 1.63 & 1.30 & 1.59 \\
KGT & 2.20 & 2.41 & 1.33 & 1.57 & 1.32 \\
GTK & 2.43 & 2.31 & 1.39 & 1.83 & 1.48 \\
GKT & 2.39 & 1.96 & 1.89 & 1.24 & 1.70 \\
TKG & 1.98 & 2.04 & 1.41 & 1.80 & 1.85 \\
TGK & 2.24 & 2.44 & 1.55 & 1.56 & 1.61 \\
Entire List & 13.31 & 12.98 & 9.20 & 9.30 & 9.55 \\
SD & 7.41 & 8.16 & 6.29 & 5.77 & 5.04 \\
\hline
\end{tabular}

$F$ ratio of 62.63 , which is significant well beyond the .01 level for 1 and $102 \mathrm{df}$, shows the enormous difference in learning rate that the difference between the two series of stimulus trigrams has caused. This bolsters the generalization concerning an inverse relationship between ISS and learning rate that has been previously supported in a large number of studies (e.g., Dey, 1966). The effect of ITI also is significant, $F(2,102)=3.84, p<.05$, while the ISS by ITI interaction is not significant. However, when analyses of variance with one-way classification are separately carried out for the two degrees of ISS, the variance for ITI turns out to be nonsignificant, $F(2,51)=1.31$ for $L S$, but significant at the .05 level, $F(2,51)=3.27$ for HS. Fisher's least significant difference (LSD) for the three HS means is 6.69 , which implies that facilitation is reliable for the 30-sec ITI but just short of the significance mark for the 60 -sec ITI. The conclusion that the effects of ITI discussed above afford is: significant facilitation by increased ITI when the stimuli are similar, but not when they are dissimilar, evidences the importance of LSS as a primary condition for DP facilitation in PA learning, though it is acknowledged that the evidence would be more viable in the event of a significant interaction effect.

Out of all incorrect responses, the overt intrusions, omissions, and extralist responses are, respectively, $53.03 \%$, $41.94 \%$, and $4.93 \%$. The group means of total overt intrusions, as well as intrusions per trial, are shown together with respective standard deviations in Table 1. Both of these data were subjected to the same analyses as the trials to criterion $6 / 6$.
It is revealed that significantly more overt intrusions have been produced by HS than by $L S, F(1,102)=34.94, p<.01$. Similar findings were earlier reported by Gibson (1942), Plotkin (1943), and Marshall \& Runquist (1962). However, the overt intrusions are reliably affected by ITI, neither when the experiment is taken as a whole nor when the two degrees of ISS are considered separately. The analyses of intrusions per trial reveal no significant effect whatsoever. This is at variance with the reports by Underwood $(1951,1953 a)$ and Feldman \& Underwood (1957) of greater per-trial intrusions under high than under low ISS and by Underwood $(1953 a, b)$ of decreasing per-trial intrusion frequency as a function of ITI.

The six HS trigrams are constructed with the same three letters so that each trigram is related to the other five trigrams in five unique ways. Using the notation 1-2-3 to signify the sequence of letters in any trigram, the relative letter sequences in the other trigrams may be designated 1-3-2, 2-1-3, 3-2-1, 3-1-2, and 2-3-1. The overt intrusions elicited by each stimulus in the performance by all the three HS groups are grouped into these five categories. The mean frequencies $(\mathrm{N}=54)$ in these categories for the six stimuli and the sums of the mean frequencies in the five categories together with standard deviations are shown in Table 2. An illustration to make the meaning of the figures in this table clear follows: The cell means in the first row signify that the mean frequencies with which KTG incorrectly elicited the responses belonging to KGT, TKG, GTK, GKT, and TGK are, respectively, $2.07,1.82,1.63,1.3$, and 1.59 .

A test by analysis of variance for the significance of difference among the five category means for the entire list has resulted in an $F$ ratio of 19.42 , which, for 4 and $212 \mathrm{df}$, is significant beyond the .01 level. The LSD at the .05 level, based upon the mean Ss by Category interaction variance of 15.47 in this analysis, is 1.52 . In the light of this value of LSD, significant difference exists neither between the Categories 1-3-2 and 2.1-3 nor among the Categories 3-2-1, 3-1-2, and 2-3-1, while each category in the former group significantly differs from every category in the latter group. The upshot of all these is that more response substitutions occur between stimulus trigrams when the position of the first or third letter is unchanged than when either the position of the middle letter is unchanged or the positions of all three letters are rearranged. DISCUSSION

Underwood \& Ekstrand (1967) manipulated ITI in three separate experiments, one for each degree of ISS. The results showed significant ITI effect in only Experiment 3, which involved high ISS. This by itself would be a solid evidence of the interrelatedness of the DP and ISS variables, were it not for the fact that the PA lists used in the three experiments were of unequal length. By producing essentially the same result with the variable of list length controlled, the present experiment proves that the interpretation of DP facilitation in terms of the dissipation of competition-induced inhibition through rest intervals is as valid when ITI is used as when IUI is used as the medium for varying DP.

Assuming that the intrusion errors are overt manifestations of intralist stimulus generalization, the conclusion drawn from the statistical analysis of the intrusion data summarized in Table 2 may be translated thus: Two stimulus trigrams composed of the same letters generalize considerably more when they have the same letter in the first or third position than when either the letters in the second position are identical or all three letters are juxtaposed. This fact may be provided a tentative explanation by a hypothesis that combines the orthodox compound-stimulus theory (Hull, 1943), according to which response association is distributed over all elements of a compound stimulus, and the Pavlov-Hull concept of perseverative stimulus trace (Hull, 1937, 1952; Hull et al, 1940). Each trigram used in this experiment is a stimulus compound and each letter a stimulus element. As the stimulus elements are exposed in succession and there is a temporal gap between the stimulus compound and the $R$ term, association is presumably formed with a trace compound composed of the segments of the stimulus elements' traces that are continuous with the $\mathrm{R}$ term. The stimulus trace being a decay function of time, the magnitude of a component trace is an inverse function of the remoteness of the stimulus element from the $\mathrm{R}$ term, so that the component traces corresponding to the elements occurring first, second, and third in the sequence have, respectively, low, medium, and high magnitudes. The six stimulus trigrams' trace compounds are all 
composed of the same traces, while differing with regard to the component traces' magnitudes. If we accept the proposition that the difference between two trace compounds is the sum total of the differences between the component traces' magnitudes, then it follows that a stimulus trigram (1-2-3) has greater similarity and therefore generalizes more with the two trigrams having the relative sequences 1-3-2 and 2-1.3 than with the rest, which have the relative sequences 3-2-1, 3-1-2, and 2-3-1.

The concept of trace compound and the notion that when two trace compounds have identical components, generalization between them is an inverse function of the aggregate magnitude difference between the identical component traces, have been utilized to interpret verbal learning phenomena in at least one other instance. Hull et al (1940) have offered an explanation of the forward and backward intralist errors in serial anticipation learning in terms of generalization between the trace compounds formed by the perseverative stimulus traces left by the syllables at preceding positions in the series. However, the validity of trace compound as an analytical device and of its use in the interpretation of stimulus generalization would essentially depend upon confirming evidences from the studies of conditioned response. To the writer's knowledge, the literature on conditioning does not offer any data by which this can be checked upon.

\section{REFERENCES}

DEY, M. K. An explanation of distributed practice efficacy in paired-associate learning. Journal of General Psychology, 1966, 74 61-75.

\section{Bizarre images in artificial memory}

GARY G. BRIGGS and STEPHEN HAWKINS, Duke University, Durham, N.C. 27706, and HERBERT F. CROVTTZ, Veterans Administration Hospital Durham, N.C. 27705

Recent work on the ancient art of artificial memory, in which first a set of places is memorized and then things or words are "put in those places with bizarre images," has shown that $S$ need not first commit a set of places to memory, but instead may successfully use a set of locations arbitrarily selected and provided by $E$. In the present study arbitrary "memory loci" and "bizarre images" are
FELDMAN, S. M., \& UNDERWOOD, B. J. $S$ timulus recall following paired-associate learning. Journal of Experimental Psychology, $1957,53,11-15$.

GIBSON, E. J. Intralist generalization as a factor in verbal learning. Journal of Experimental. Psychology, 1942, 32, 185-200.

HULL, C. L. Mind, mechanism and adaptive behavior. Psychological Review, 1937, 44, 1.32 .

HULL, C. L. Principles of behavior. New York: Appleton-Century-Crofts, 1943.

HULI, C. L. $A$ behavior system. New Haven: Yale University Press, 1952.

HULL, C. L., HOVLAND, C. I., ROSS, R. T. HALL, M., PERKINS, D. T., \& FITCH, F. B. Mathematico-deductive theory of rote learning. New Haven: Yale University Press, 1940. Facilitation in paired associate learning by distributed practice. Journal of Verbal Learning \& Verbal Behavior, 1962, 1, 258-263.

PLOTKIN, L. P. Stimulus generalization in Morse code learning. Archives of Psychology, 1943. 40 , No. 287.

UNDERWOOD, B. J. Studies of distributed practice: II. Learning and retention of paired-adjective lists with two levels of intralist similarity. Journal of Experimental Psychology, 1951, 42, 153-161.

UNDERWOOD, B. J. Studies of distributed practice: Vill. Learning and retention of paired nonsense syllables. Joumal of Experimental Psychology, 1953a, 45, 133-142.

UNDERWOOD, B. J. Studies of distributed practice: IX. Learning and retention of paired adjectives as a function of intra-list similarity. Journal of Experimental Psychology, 1953b, 45, 143-149.

UNDERWOOD, B. J., \& EKSTRAND, B. R. Effects of distributed practice on paired-associate learning. Journal of Experimental Psychology Monograph Supplement, 1967, 73(4, Whole No. 634).

\section{NOTE}

1. All requests for reprints should be mailed to P.o. Box 36, McMaster University, Hamilton, Ont., Canada
MARSHALL, M. A., \& RUNQUIST, W. N

with them. Crovitz (1969) has shown that Ss can use a set of arbitrary locations in the place of self-produced memory loci and can use them in forming "bizarre images" as mediators in one-trial paired-associate learning. Delin (1968) reported that the degree of "bizarreness" of mediating "images" is positively correlated with retention in PA learning, and Bugelski, Kidd, \& Segmen (1968) have reported that the time that is available for the "construction of the image" is a critical variable determining the effectiveness of the mnemonic technique.

The value of any mnemonic technique that facilitates retention and recall must depend upon the time and effort required for the successful use of the technique. The self-production of memory loci is not essential, as has been indicated; the present study was designed to determine whether self-production of "bizarre images" is itself essential.

A set of 20 fictitious locations along an artificial "map of Gorky Street" was drawn on a classroom blackboard, the 20 successively numbered locations corresponding to those used by Crovitz (1969). A set of 20 common, picturable English nouns had been randomly selected from the vocabulary of The System of Basic English (Ogden, 1934) and, prior to the experimental session, the authors, who are experienced in the classical art of artificial memory, fashioned sentences corresponding to "bizarre" mediators between the locations and the English nouns.

The Ss were 50 undergraduates, who participated as a course requirement, tested in a group. They were instructed: "This is an experiment involving the use of an artificial memory device. I will read you a list of words and give you an association between each word and the corresponding location on the blackboard. After I finish, write on your paper the list of words you were given, pairing them with the appropriate location. For example, BATHTUB: Imagine a whale in a bathtub. The word is WHALE. You would write the word WHALE at the number corresponding to the location BATHTUB. Now number your sheet from 1 to 20 .

1. ELECTRIC COMPANY. Picture a plow cutting an underground cable. The word is PLOW. (88\%)

2. GAS STATION. Picture the attendant angrily driving a nail into your tire. The word is NAIL. (94\%)

3. OCCULIST. Picture making the As described by Yates (1966), the ancient art of artificial memory required the storage in memory of a sequence of occulist mad, causing him to stick his locations and then the formation of finger in your eye. The word is FINGER. "bizarre images" as mediators between the (92\%) locations and the items to be associated 